
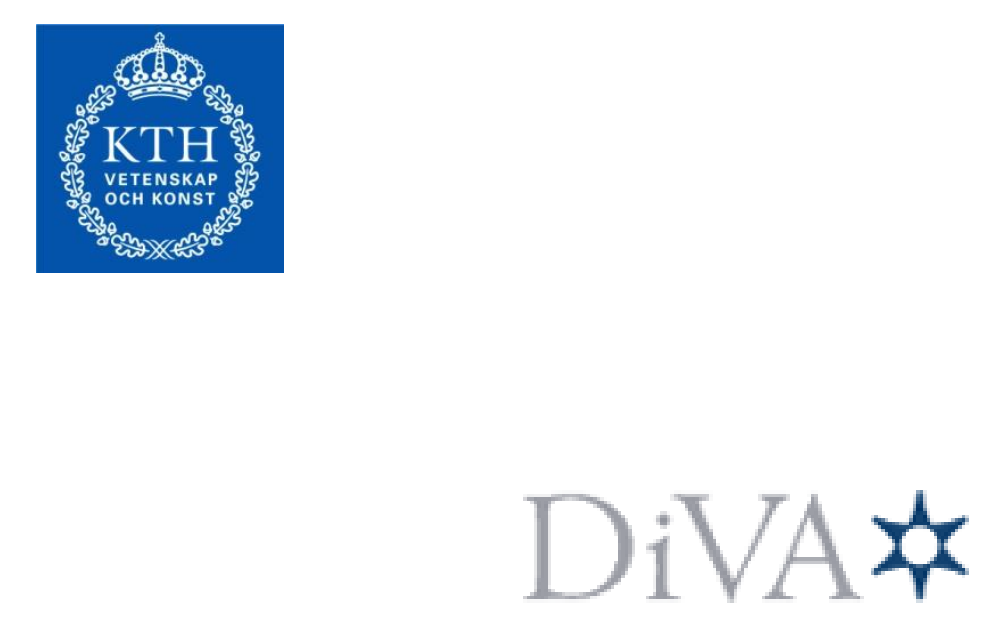

http://kth.diva-portal.org

This is an author produced version of a paper published in Swedish Communication Technologies Workshop (Swe-CTW), 2012.

This paper has been peer-reviewed but does not include the final publisher proofcorrections or proceedings pagination.

(C) 2012 IEEE. Personal use of this material is permitted. Permission from IEEE must be obtained for all other uses, in any current or future media, including reprinting/republishing this material for advertising or promotional purposes, creating new collective works, for resale or redistribution to servers or lists, or reuse of any copyrighted component of this work in other works.

Citation for the published paper:

Amin, Shoaib, Efrain Zenteno, Per N Landin, Daniel Rönnow, Magnus Isaksson, Peter Händel.

Noise Impact on the Identification of DigitalPredistorter Parameters in the Indirect LearningArchitecture.

Swedish Communication Technologies Workshop (Swe-CTW), 2012.

Access to the published version may require subscription.

Published with permission from: IEEE 


\title{
Noise Impact on the Identification of Digital Predistorter Parameters in the Indirect Learning Architecture
}

\author{
Shoaib Amin*†, Efrain Zenteno*†, Per N. Landin*†, Daniel Rönnow*, Magnus Isaksson*, and Peter Händel ${ }^{\dagger}$ \\ * Dept. Electronics, Mathematics, and Natural Sciences, University of Gävle. 80176 Gävle. \\ $\dagger$ ACCESS Linnaeus Centre, Signal Processing Lab, KTH Royal Institute of Technology, 10044 Stockholm.
}

\begin{abstract}
The indirect learning architecture (ILA) is the most used methodology for the identification of Digital Pre-distorter (DPD) functions for nonlinear systems, particularly for high power amplifiers. The ILA principle works in black box modeling relying on the inversion of input and output signals of the nonlinear system, such that the inverse is estimated. This paper presents the impact of disturbances, such as noise in the DPD identification. Experiments were performed with a state-of-art Doherty power amplifier intended for base station operation in current telecommunication wireless networks. As expected, a degradation in the performance of the DPD (measured in normalized mean square error (NMSE)) is found in our experiments. However, adjacent channel power ratio (ACPR) can be a misleading figure of merit showing improvement in the performance for wrongly estimated DPD functions.
\end{abstract}

\section{INTRODUCTION}

The "green" trend drives technology towards increased efficiency also in telecommunication. Simultaneously, the requirements from the customers in terms of capacity increases every year with the increasing number of mobile devices. These two simultaneous demands put more and more stringent requirements on the components handling the signals. One such component that is required in all wireless communication is the radio frequency (RF) power amplifier (PA).

The RF PA is the active component creating the highest power levels in any wireless communication system, which means that it has to operate with high efficiency in order not to waste supplied power. On the other hand, highly efficient operation of a PA when using amplitude modulated signals typically results in nonlinear distortions [1] that degrade the performance of the network. For this reason, regulatory requirements limit the amount of distortion that is tolerable, commonly in the form of limits on the power emitted in neighboring frequency channels.

Due to these requirements it has become necessary to improve the linearity and thereby reducing the distortions. One such method is that of digital pre-distortion (DPD). A commonly used method to find the parameters of the DPD is the indirect learning architecture (ILA) [2]. The ILA is based on identifying a post-distorter that is used as a pre-distorter. The advantage of the ILA is that the computational burden is considerably reduced compared to $P$ :th order inverses in the case of Volterra systems or direct learning approaches [2]. However, the ILA requires an inversion of a system where the

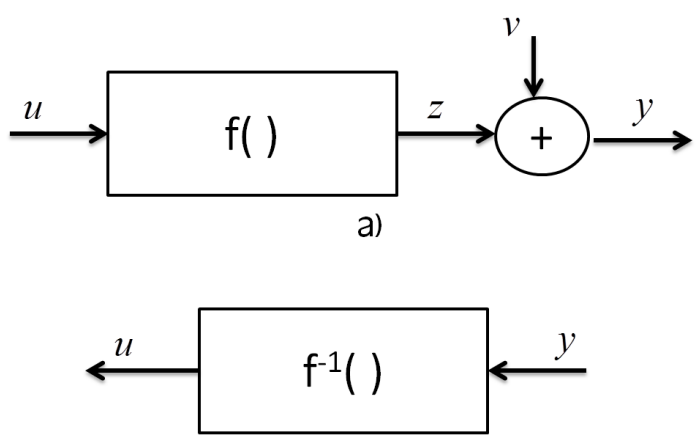

b)

Fig. 1. ILA for inverse identification.

noise is inside of the regression matrix, a nonlinear error-invariables problem [3]. This effect is investigated in this paper.

The structure of the paper is as follows. Section II introduces the ILA, the modeling, and shows the derivation of the bias in model parameters due to the noise. The experimental set up is described in section III. Results and discussion are given in section IV and the conclusions are presented in section V.

\section{THEORY}

This section introduces DPD, the ILA and derives the bias terms due to the noise when using a linear least-squares estimation procedure.

\section{A. DPD and the ILA}

The principle of DPD parameter estimation using the ILA is depicted in Fig. 1. The output signal $y$ of the device under test (DUT) was measured. The input signal $u$ is known and is considered to be noise free, as illustrated in Fig. 1 a) and b). the input and output signals are used to identify the inverse system using the ILA principle [2], where the input is treated as output and the output as input.

In a more realistic scenario, the measurement process will add noise to the sequences [4]. Thus, the output signal (denoted $z$ in the Fig. 1 a) will be polluted by a noise sequence $(v)$ to render the measured output $(y)$. This creates the nonlinear error-in-variables problem to be studied in this paper. 


\section{B. Modeling}

To estimate the behavior of a system from input and output measurements is known as system identification. The identification process involves various issues such as modeling system structure, model order, estimation of model parameters and its validation [5]. In this paper the behavioral model used for the identification is extended envelope memory polynomial (EEMP) [6]. EEMP is described by the following equation

$$
y(n)=H_{1}+H_{2},
$$

with

$$
H_{1}=\sum_{m_{1}=0}^{M_{1}} h_{m_{1}} u\left(n-m_{1}\right)
$$

and

$$
H_{2}=\sum_{p=2}^{\left[\frac{P+1}{2}\right]} \sum_{m_{2}=0}^{M_{2}} h_{m_{2}}^{2 p-1} u(n)\left|u\left(n-m_{2}\right)\right|^{2(p-1)}
$$

where $M_{1}$ and $M_{2}$ are two separate memory depths, $\mathrm{P}$ is the model order, $h_{m_{1}}$ and $h_{m_{2}}$ are the model coefficients. EEMP is an extension of the envelope memory polynomial (EMP) [6] which includes linear memory terms to encounter the frequency dependency of the linear term.

\section{Identification}

The estimation of the model parameters from a set of inputoutput measurements is performed by minimization of the sum-squared error between the observed data and the model output:

$$
J(\theta)=\sum_{n=0}^{N-1}|e(n)|^{2}=\sum_{n=0}^{N-1}|y(n)-f(u(n), \theta)|^{2} .
$$

In (4), $J(\theta)$ defines the cost function to be optimized, $u(n)$ and $y(n)$ are the $n: t h$ sample of the input and output signals, respectively, and $N$ the total number of samples. $f(u(n), \theta)$ is the nonlinear function describing the input to the output relationship.

The model in (1) is linear in the parameters. Therefore, the problem of estimating the parameters $\theta$ can be rewritten in matrix form as

$$
J(\theta)=\|\mathbf{y}-\mathbf{H} \theta\|_{2}^{2},
$$

where $\mathbf{H}=\mathbf{H}(\mathbf{u})$ is the regression matrix. Hence, the least square estimation (LSE) criterion is employed to find this estimate as:

$$
\widehat{\theta}=\left(\mathbf{H}^{*} \mathbf{H}\right)^{-1} \mathbf{H}^{*} \mathbf{y} .
$$

For the case presented in this paper, input and output are interchanged in equation 1 accordingly to the ILA principle for identifying the inverse system.

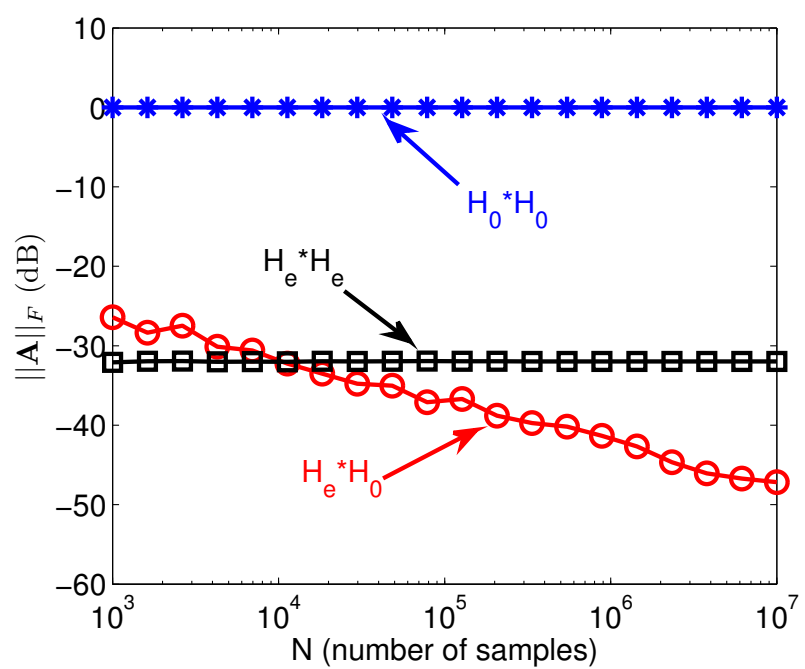

Fig. 2. Frobenius norm of the covariance and cross-covariance matrices as a function of the number of samples $\mathrm{N}$.

\section{Noise Impact}

As described in a previous section, the identification is made using LSE techniques (QR decomposition for example in Matlab) by solving:

$$
\widehat{\theta}=\underset{\theta}{\operatorname{argmin}}\left\{\|\mathbf{u}-\mathbf{H} \theta\|_{2}^{2}\right\} .
$$

With $\theta$ containing the parameters of the inverse model. The regression matrix formed by the output sampled sequence is polluted with additive white Gaussian noise (AWGN) of zero mean and $\sigma^{2}$ variance. Hence the regression matrix gets a contribution from the noise that is model dependent such a contribution is denoted by $\mathbf{H}_{e}$ in the following equation:

$$
\mathbf{H}=\mathbf{H}(\mathbf{y}+\mathbf{v})=\mathbf{H}_{0}+\mathbf{H}_{e} .
$$

Using LSE in equation (8) yields:

$$
\widehat{\theta}=\left(\left(\mathbf{H}_{0}+\mathbf{H}_{e}\right)^{*}\left(\mathbf{H}_{0}+\mathbf{H}_{e}\right)\right)^{-1}\left(\mathbf{H}_{0}+\mathbf{H}_{e}\right)^{*} \mathbf{u} .
$$

Note that the matrix inverse in the right hand side of equation (9) contains the terms $\mathbf{H}_{e}^{*} \mathbf{H}_{0}$, and $\mathbf{H}_{0}^{*} \mathbf{H}_{e}$. These terms represent the cross covariance matrices of output of the model and disturbance. Since the noise is uncorrelated with both the input and output sequences, for a number of samples $(N)$ that goes to infinity, the covariances will tend to zero. A simulation of the DPD identification with an output sequence polluted with AWGN yields the results shown in Fig. 2. The signal-to-noise-ratio (SNR) is set to $28 \mathrm{~dB}$ and the covariance and cross covariance matrices are estimated for several number of samples and normalized to $\mathbf{H}_{0}^{*} \mathbf{H}_{0}$. Fig. 2 shows the Frobenious norm of respective matix (in $\mathrm{dB}$ ) for different number of samples. It is clear that, the crosscovariance matrix reduces its Frobenious norm with a trend towards zero. 


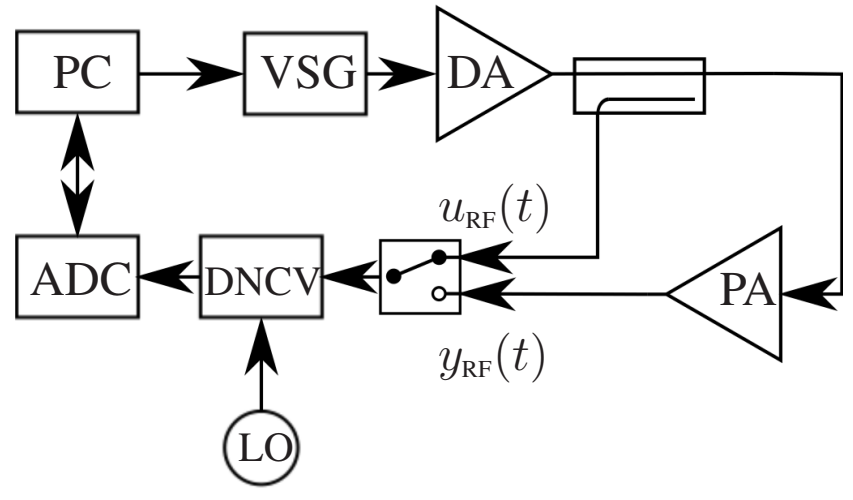

Fig. 3. Measurement setup to assess the impact of noise in the DPD identification using the ILA principle.

Hence, for large $N$ the cross-covariance matrices $\mathbf{H}_{e}^{*} \mathbf{H}_{0}$ and $\mathbf{H}_{0}^{*} \mathbf{H}_{e}$ can be neglected. Thus, equation (9) can be written as:

$$
\widehat{\theta}=\left[\mathbf{H}_{0}^{*} \mathbf{H}_{0}+\mathbf{H}_{e}^{*} \mathbf{H}_{e}\right]^{-1}\left(\mathbf{H}_{0}+\mathbf{H}_{e}\right)^{*} \mathbf{u}
$$

Using the well known inverse matrix lemma, the last expression can be expanded in:

$$
\widehat{\theta}=\left(\mathbf{H}_{0}^{*} \mathbf{H}_{0}\right)^{-1} \mathbf{H}_{0}^{*} \mathbf{u}+B
$$

with

$$
B=\mathbf{Y u}-\left\{\mathbf{X}[\mathbf{I}+\mathbf{X}]^{-1}\left(\mathbf{H}_{0}^{*} \mathbf{H}_{0}\right)^{-1}\right\}\left(\mathbf{H}_{0}^{*}+\mathbf{H}_{e}^{*}\right) \mathbf{u} .
$$

Previous equations show that the estimated $\widehat{\theta}$ contains a bias term $(B)$ which depends on the noise level. With the matrices $\mathbf{X}$, and $\mathbf{Y}$ denoting

$$
\begin{aligned}
& \mathbf{Y}=\left(\mathbf{H}_{0}^{*} \mathbf{H}_{0}\right)^{-1} \mathbf{H}_{e}^{*} \\
& \mathbf{X}=\left(\mathbf{H}_{0}^{*} \mathbf{H}_{0}\right)^{-1}\left(\mathbf{H}_{e}^{*} \mathbf{H}_{e}\right) .
\end{aligned}
$$

\section{EXPERIMENTAL}

The measurement setup is shown in Fig. 3. It consists of a vector signal generator (VSG), a driver amplifier, the PA under test, a wideband downconverter and an analog-to-digital converter (ADC) with a sampling rate of $400 \mathrm{MHz}$ and a resolution of 14 bits. The sampling frequency was chosen to capture an exact number of points for every measured period, known as coherent sampling [7].

Test signals were $5 \mathrm{MHz}$ wide random multitone signals with crest factor of approximately $10 \mathrm{~dB}$. Two signal sets were used, one for identification of the predistorter parameters and one for the validation.

Performance results are presented in terms of normalized mean-square error (NMSE) and as adjacent channel power leakage ratio (ACPR). The NMSE is given by

$$
\mathrm{NMSE}=\frac{\int \Phi_{e}(f) d f}{\int \Phi_{y}(f) d f}
$$

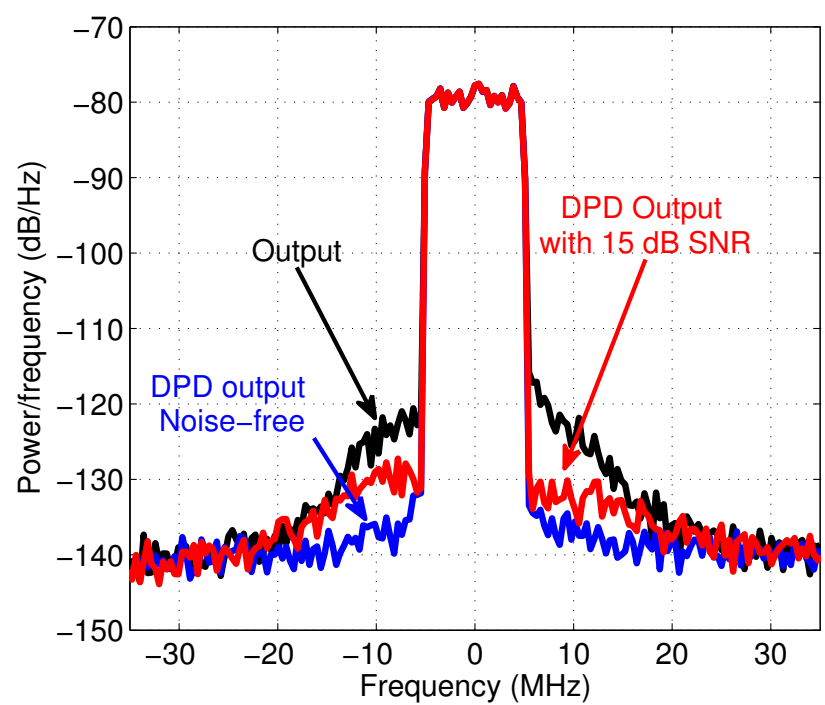

Fig. 4. Power spectrum of the output and DPD output of the PA. Two cases shown, SNR $=15 \mathrm{~dB}$ and noise-free for identification.

where $\Phi_{e}(f)$ is the power spectrum of the model error $e(n)$ in (4) and $\Phi_{y}(f)$ is the power spectrum of the measured output signal $y(n)$. The integration is carried out across the available bandwidth. The ACEPR is given by

$$
\text { ACEPR }=\frac{\int_{\text {adj. ch. }} \Phi_{e}(f) d f}{\int_{\text {ch. }} \Phi_{y}(f) d f},
$$

where the integration in the numerator is done over the adjacent $5 \mathrm{MHz}$ wide channel with the largest amount of power and the integration in the denominator is done over the input channel band.

The PA under test is a Doherty amplifier intended for $3 \mathrm{G}$ operation. In the experiments, this DUT was not operated in extreme compression; since models usually show lower performances in this regimes. Our aim is to depict the noise impact on the DPD parameter identification. Hence, the amplifier was operated under mild saturation conditions and the model used for it has shown good performances.

\section{RESUlts}

The measurement step was performed with coherent averaging to minimize the impact of the measurement system, a coherent averaging of 800 times is performed in the measured sequences. Such process reduces the level of the noise floor of the VSA. In a subsequent step, the measured output identification sequence was digitally contaminated with complex circularly symmetric Gaussian noise at certain SNR to further proceed with the identification of the DPD parameters as described in Section II. As shown earlier in this paper, the noise polluting the output sequence creates a bias estimate of the DPD coefficients. Further, such a bias depends on the DPD model as well as on the SNR conditions of the experiment. 


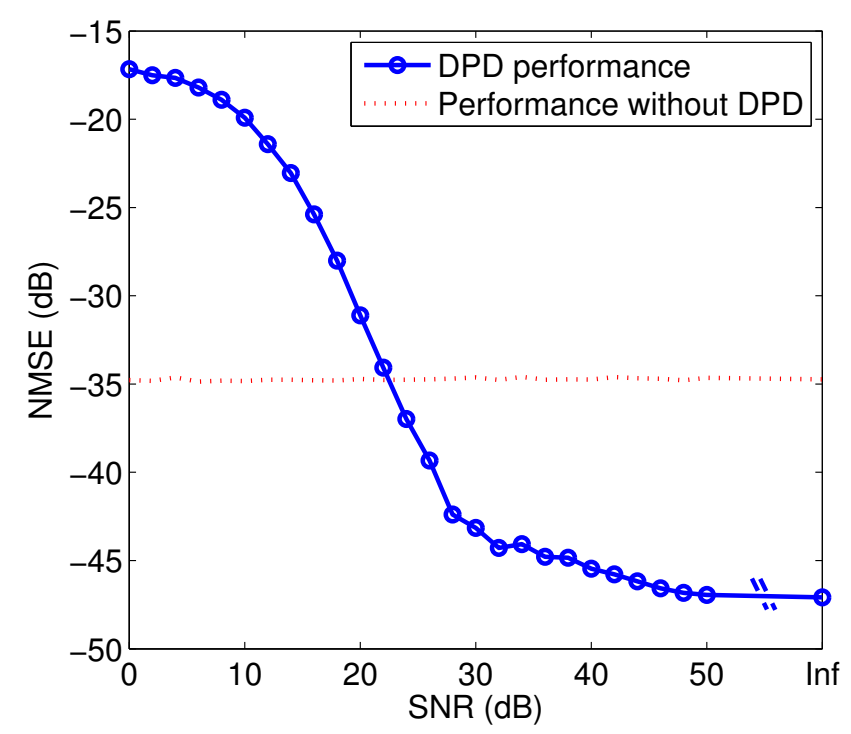

Fig. 5. DPD performance (measured in NMSE) under different SNR conditions in the identification.

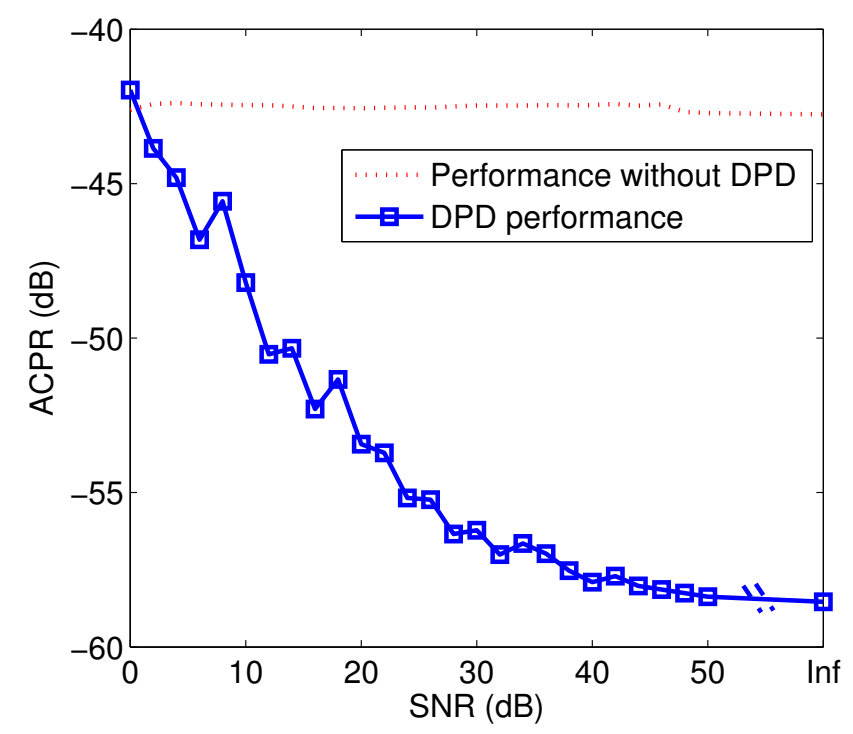

Fig. 6. DPD performance (measured in ACPR) under different SNR conditions in the identification.

Fig. 4 shows the power spectrum of the output (no compensated) and the DPD output for two levels of noise in the identification of the DPD.

Fig. 5 shows the NMSE obtained with DPD for different SNRs in the identification output sequence. The NMSE level obtained without DPD is also shown in the Fig.5.

Fig. 6 shows ACPR results with and without applying DPD for different SNR conditions in the identification. This result indicates for lower SNRs, e.g. lower than $25 \mathrm{~dB}$, the performance of DPD as a measure of ACPR has improved as shown in Fig. 6.

Fig. 5 indicates that NMSE degrades when noise is present in the measured data, such degradation can worse the performance compared to the case where no DPD is applied (SNR $\approx 23 \mathrm{~dB}$ ).

On the other hand, ACPR shows a better performance which can be a misleading result if only this metric is chosen as a figure of merit for analysis of the performance.

\section{CONCLUSION}

DPD identification using the ILA principle with noisy output sequences results in a bias estimate when using LSE solution. Such bias degrades the DPD performance measured as NMSE and ACPR. As pointed in Fig. 5 and Fig. 6, the ACPR can be a misleading figure of merit for assessing the DPD performance of the DPD function in the presence of noise.

\section{REFERENCES}

[1] S. Cripps, Advanced Techniques in Rf Power Amplifier Design. Upper Saddle River, NJ, USA: Artech House, 2002.

[2] C. Eun and E. Powers, "A new volterra predistorter based on the indirect learning architecture," IEEE Trans. Signal Process., vol. 45, no. 1, pp. $223-227$, jan 1997.

[3] L. Ljung, System identification: theory for the user. Upper Saddle River, NJ, USA: Prentice-Hall, Inc., 1986.

[4] D. Wisell, D. Ronnow, and P. Handel, "A technique to extend the bandwidth of an rf power amplifier test bed," IEEE Trans. Instrum. Meas., vol. 56, no. 4, pp. 1488 -1494, Aug. 2007.

[5] P. N. Landin, M. Isaksson, and P. Händel, "Parameter extraction and performance evaluation method for increased performance in $\mathrm{rf}$ power amplifier behavioral modeling." Int. J. RF. Microw. Comp. Aid. Eng., vol. 20, pp. 200-208, Mar. 2010.

[6] P. Landin, "Digital baseband modeling and correction of radio frequency power amplifiers," Ph.D. dissertation, Electrical Engineering, The Royal Institute of Technology, KTH. Vrije Universiteit Brussels., 2012.

[7] "IEEE standard for terminology and test methods for analog-to-digital converters," IEEE Std 1241-2010 (Revision of IEEE Std 1241-2000), pp. $1-139$, Jan. 2011. 